\title{
A Framework for Modeling and Designing of Intelligent and Adaptive Interfaces for Human Computer Interaction
}

\author{
Syed Imran Ali \\ Asst. Professor, \\ CSE, TITR, \\ Bhopal, India
}

\author{
Sameer Jain \\ Asst. Professor, IT \\ MPSTME, NMIMS, \\ India
}

\author{
Banshi Lal \\ Patidar \\ Asst. Professor, IT, \\ TITR, Bhopal, \\ India
}

\author{
Neeraj Sharma \\ Asst. Professor, IT, \\ TITR, Bhopal, \\ India
}

\begin{abstract}
This Paper presents a framework for modeling and designing of intelligent and adaptive interfaces for human computer interaction. Since interfaces along with their interaction styles have got a vital role to play in mass and massive adoption of technology therefore timely we must look for and explore next generation multimodal, adaptive and intelligent interfaces and interaction styles enriched with good expression capabilities and natural characteristics to achieve higher user acceptance, usability and satisfaction level .This can only be achieved by empowering and enriching human computer interaction and interfaces along with its associated components.
\end{abstract}

\section{General Terms}

Modeling, designing, interfaces for human computer interaction

\section{Keyword}

Intelligent and adaptive interfaces, human computer interaction, user acceptance, usability and satisfaction level.

\section{INTRODUCTION}

Today, one of the most challenging tasks is to transform the concept "Technology-based Computing Systems for Everyone and for Every Use at any Place and at any Time" into reality. In spite of rapid advancements in this IT field, its overall penetration and acceptability into our lives is not up to that extent as it was earlier expected. In the latest era of Ubiquitous Computing and intelligent space [01], when almost invisible intelligent computing devices equipped with fine quality miniaturized sensors and highly diversified interaction styles, connected in a high bandwidth network are ready to help us in our mundane tasks to working lives for enormously growing range of purposes, it is quite evident that prevailing WIMP (Window, Icon, Menu and Pointing Device) graphical user interfaces along with present interaction styles are no longer sufficient and competent to cater the requirements, expectations of technology users [02]. These technologies and devices don't necessarily result in mass user acceptance and satisfaction Human computer interaction is a multidisciplinary field which encompasses computer science, cognitive science, sociology, psychology, anthropology, philosophy, and ergonomics etc [07]. The Special Interest Group on Computer- Human Interaction (SIGCHI) of the Association of Computer Machinery (ACM) defines Human Computer Interaction (HCI) as a discipline concerned with design, evolution, implementation of interactive computing systems for human use, and with the study of major phenomena surrounding them [08].

It is the interface and interaction style of a system which enables the users to interact/communicate with system, lets him/her use it successfully and accomplishing the intended task. Different roles that these interfaces play are as follows:

(i) Service Access Point: Mediate between user and computer and let the users successfully accomplish their intended task by using the offered services with maximum satisfaction and trust in the system.

(ii) Act as an interpreter between user's goal and inputs.

(iii) Provide feedback and output to the user as per their expectations and mental models.

Today not all but most of computer programs, application software and their interactive interfaces are apparently complex, hard to understand and use effortlessly. While the prime objective of these user interfaces is to make the user's interaction with the computational system as optimum as possible, many times even an experienced professional user bumps into problem during interacting with a computer, can't make full use of system and does not enjoy the interaction at all due to ill-designed interfaces. These problems may arise due to many reasons $[04,09,10]$ for example

- Use of confusing menu options, wrong order grouping and spatial placement, inappropriate and nonstandard terminology, icons or incomprehensive dialogue boxes or error reports/messages.

- Improper and inconsistent use of colors, sound, icons, space, video etc. which doesn't fit with human cognition.

- Use of illegible texts, indiscernible or similar looking objects in display.

- Lack in ability to personalize, adapt the interfaces as per the user and lack of intelligent decision aid.

- Use of few modalities for interaction which does not take advantage of super computational power of today's processor.

These deficiencies slowly but surely lead towards user inconvenience, disruption and dissatisfaction. As a result usability, acceptability and user satisfaction of the system hampers adversely. Therefore, definitely there is a need of enhanced, intelligent and adaptive next-generation interfaces enriched with many more attributes.

Human Computer Interaction and Interface Design is focal research and development area in innovative multidisciplinary 
computing regime. It not only posses many socio-technical challenges but also offers enormous opportunities related with specifying, designing and implementation of the interactive interfaces to interaction designers, architects and researchers who may have mixed set of knowledge and expertise from different streams like psychology, sociology, cognitive science, marketing, information systems, business, ergonomics, entertainment, graphic design, art, computer science etc. The development of humanization operation makes human-computer interaction become more natural and friendly [11]. Any Intelligent and Adaptive Interface possesses the ability to

1. Personalize as per user's characteristics.

2. Recognize different contexts/plans and extend situational aid to user.

3. Make use of multiple modalities for communication with the user.

4. Respond to the user well in comply with the mental model and expectations of the user (this includes the affective aspects of the user).

5. Learn continuously and adapt as per the situation/ environment.

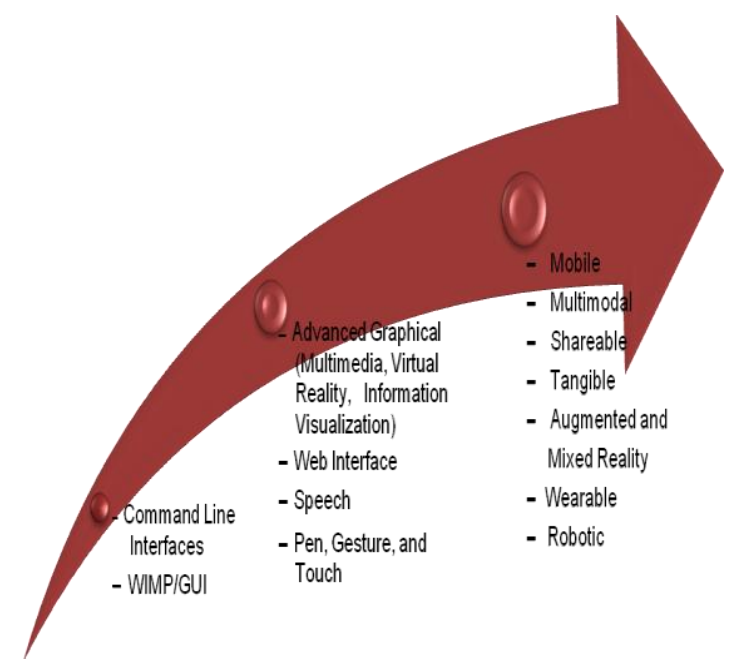

Figure 1- Evolution of User Interfaces

\section{A BRIEF REVIEW OF THE WORK ALREADY DONE IN THE FIELD}

Initially in early 1980s, four different streams [10] namely (i) Software development life cycle and models from software engineering (ii) software psychology and ergonomics in computing systems (iii) computer graphics and user interfaces (iv) models, theories and frameworks from cognitive science; converged as a result of initiatives taken by some leading organizations like ACM and IFIP including the setup of Special Interest Group by ACM and Task Group by IFIP on Compute Human Interaction. Further HCI started to take shape up as multifaceted core discipline around in 1982 when in USA National Bureau of Standard conference on "Human Factors on Computer Systems" was conducted. About the same time many more relevant conferences were organized throughout the world and as a result quality literature started to appear.
Being most of the applications of HCI allied with Computer Science, the research and development of HCI had become very well integrated in Computer Science in late 80s. This was more strengthening in 1988 when ACM task force declared HCI as one of the nine core areas of Computer Science [06]. The first Handbook of Computer Science and Engg.(1997) too included HCI as one of the ten major sections.

The importance and need of quality user interfaces was first considerably realized in 1980's decade and as a result command line and WIMP (Window, Icon, Menu and Pointing Device) interfaces were developed. Since then to present time these user interfaces have gone through radical changes in their designs, appearances, usability, utility, functionalities and services to users etc. The advancements of information technology along with faster hardware devices and superior software resulted in increased penetration of it onto our daily and working lives. Therefore variety of user interfaces are available today ranging from traditional object oriented desktop, multimedia and web interfaces to particular application specific interfaces like wearable, robotic, remote operated, mixed reality interfaces.

Significant work has been done to have these abilities in the interactive interfaces but still a long way to go because of constantly changing scenario of HCI. Here is review of the some of the papers.

In user modeling technique, individual user profiles are generated and grouped appropriately [04]. Each user model represents and contains information about a group of users which share common traits like similar type of personality, emotional states, habits, preferences, objectives, interests etc. After collecting the knowledge and information about user it is transformed into formats in which it can be stored in computer memory and later can be used efficiently by Inference Engine for making new inferences and learning. Predicate Logic, Baysian Networks, Scripts, Fuzzy Logic, Semantic Networks, Frames etc are frequently used knowledge representation schemes. The Inference Engine (equipped with efficient inference making algorithm like resolution for predicate logic) processes this information/ knowledge and deduces new information about the user. This learning is reflected in future interactions with the user.

The primary fact of HCI is that the user performs the task using computers. In UCD [04] the iterative design and development process always keeps the perspective user, usage situations and their characteristic at top. Thorough interviews and questionnaire sessions and observational studies are conducted for information gathering, analyzing and evaluating activities. The entire process focuses upon requirements, expectations, characteristics and limitations of perspective users at each stage of development. The user requirements and priorities can also be captured by envisioning the use of the proposed system and examining hypothetical interaction of it with perspective users [12]. The paper [13] discusses various techniques for user requirements specifications.

The interfaces designed with UCD approach are definitely much more usable, useful, need less training sessions, help and manuals [03].

In their paper [14], B. A. Goodman and D. J. Litman proposed that plan recognition can be performed by considering a user plan as schema. A library of plan schemas to represent different user plans is constructed. Each plan schema has a 
header and a parameterized description of that plan. This description consists of preconditions, decompositions, effects and constraints. For user plan/context recognition, the graphmatching algorithm of Kantz was modified and applied. In this paper CHECS (CHemical Engineering CAS System) that is a plan-based process design system was developed. Using this it was established that the plan/context recognition leads to numerous benefits for goal-oriented user behavior in areas of automatic plan completion, advice generation, Error detection and recovery and context dependent responses.

In [02,15], the HMM (Hidden Markov Model) was used for user activity recognition. Paper [02] used the definition of the context given by Dey [16] and introduced HMM for dynamic posture inference scheme using an acceleration sensor embedded in mobile handsets. It also presents two perspective applications of this approach namely E-coaching and context aware navigation application. In [15], parallel layered HMM approach was introduced for context-awareness/ recognitions for hospitals and the results of this approach were compared with other pattern-recognition algorithms like neural networks. The method uses location, identity, time and activity as contextual parameters.

Kenichi Yoshida [17] presented a framework for predicting the user commands using graph-based induction in his paper. In the proposed framework user command history is converted into colored directed graph and Markov model was used to simulate user behavior. This framework also takes the data dependency between the user commands into account. To implement this framework the UNIX operating system was modified.

The paper [18] by M. Sutterer, O. Droegehorn and K. David proposed a user profile ontology that a context sensitive adaptive application for mobile communication and information services can use to automatically trigger the situation-dependent personalization of services. This ontology allows having easy specifications of situational conditions and situation-dependent user sub-profiles.

The paper [19] by F. Arai, T. Fukuda, Y. Yamamoto, T. Naito and $\mathrm{T}$. Matsui propose a design and realization method for the intelligent adaptive interface using recursive Fuzzy reasoning. Proposed system change the automation/adaptation level in accordance with user's task performance and mental psychological state. Here the equations of recursive fuzzy reasoning are used to calculate the assistance level under the historical change of the measurement data. As an example air hockey game was discussed in virtual reality simulated environment. The statistical analysis of experimental results revealed that the proposed adaptive interface was very effective.

Since speech and images are richest sources of information exchange in any of the communication process therefore acoustic and visual recognition and their proper integration is very important in adaptive and cooperative multimodal interaction. In their paper [20] C.Bauckhage, J. Fritsch, K. J. Rohlfing, S. Wachsmuth and G. Sagerer introduced an adaptive and cooperative assembly system which performs the following task.

1. Speech recognition and understanding using HMM and knowledge-based speech understanding methods.

2. Resolve repairs and extrapositions using LR(1) parsing based unification algorithm.
3. Visual object and assembly recognition using semantic networks.

4. Monitoring and executing assembly operation using rulebased algorithm.

5. Integration of the results from speech and image under probabilistic Bayesian network.

As per the paper [21] by A. Pirhonen, it is not only inputs that any multimodal system is expected to receive from multiple modalities but the output can also be presented to user through various modalities and here the redundancy plays a vital role. If a single message from the computer is presented to the user in multiple forms then chances of misunderstanding, unwanted confusions and misinterpretation gets reduced. Moreover, if any noise is introduced during propagation by one channel then other channels will propagate and deliver the correct noise free message to user. This type of system may also be useful for users with disabilities. The redundancy not only compensates for the deficiency of the communication channel but it also secures the communication and makes the entire system more trustworthy.

As we all know that ability to recognize emotion is one of the hallmark of emotional intelligence. In the paper [22] R. W. Picard developed a system to recognize 8 emotions namely Anger, Hate, Grief, Platonic-Love, Romantic-Love, Joy, Reverence and no-emotion. It uses 5 psychological features namely electro gram, blood-volume pressure, heart-rate, skinconductivity and respiration to gather good affect data. A feature based recognition algorithm (SFFS-FP, Sequential Floating Forward Search-Fisher Projection) was proposed and compared with other algorithms in this paper. This algorithm results in $81 \%$ accuracy.

N. Okada, K. Inui and M. Tokuhisa [23] established an architectural foundation of integrated intelligent agents in their paper. This paper also presents a computational model of mind. The agent model proposed in the paper integrates the seven mental and two physical faculties namely recognition, planning, action, desire, emotion, memory, language and sensor, actuator. It first describes recognition, action and planning and then addresses their interaction while considering planning at center. This also includes image understanding. Then it discusses about actions and dialogue processing. Finally it addresses for emotion recognition. At last this paper presents an emotional dialogue subsystem as an application of affective communication.

The concept of persuasive interface is very new and challenging in HCI arena. In the paper [24] H. O. Kukkonen and $\mathrm{M}$. Harjumaa reviewed the attitude change theories from social psychology recognizing dimensions and approaches. A practical conceptual framework for understanding and designing persuasion in information system was proposed.

In [25] E. Chang, T. Dillon, D. Calder presented an overview of evolution of Human Computer Interaction and Human System Interaction in last 30 years. This paper presented the mega trends in the ICT and its use. These mega trends are: Tsunami of data, Ubiquitous digital living, Web 2.0, 3.0..., What human can do etc. The concept of Confident Computing was also introduced in paper which refers to building of the system that empower human through ambient intelligence. The importance of usability was also highlighted. This paper pointed out the paradigm shift from what computer can do for us to what human can do with computers. 
An architecture named Psychodynamic Cognitive Construction (PCC) for artificial systems to develop affective attitudes from the past history of human-machine interaction is presented in [26]. As an implementation of PCC architecture qViki system was developed. A growing network was devised to memorize and retrieve its past experiences. The preliminary experiment exhibited how the system's attitude towards certain stimulus is elicited and regulated by interaction. This paper also discussed the role that emotion may play in affective agents as a motivation system to facilitate cognitive development through social interaction.

The key challenge is to discern the most appropriate techniques and models for generating the futuristic interactive interfaces which would be quite different in characteristics like their controls, display etc. from their present GUI counterparts. This work aims to understand the needs, demands and expectations of the users of technological systems, then to propose architecture of intelligent and adaptive future multimodal interfaces to incorporate their perceptions, responses, preferences, interaction behavior etc. This work will also address the design principles, concerned issues and approaches that must be taken into consideration during specifying, designing, developing and implementing the interfaces of technology based systems in order to have effective, longer and deeper interactions. Any interactive computational system developed with these principles and approaches will definitely be able to spawn much broader and intelligent class of system responses and moreover the entire system will assist as cooperative partner to the users in accomplishing their intended tasks.

Enough literature, standards, set of thumb rules, protocols and heuristic approaches are available for incorporating some of the principles like User centered design, Real time natural look and feel in displays, Audio-visual Comforts, Good quality of information, data and easy access to the functionalities offered. Unfortunately interfaces with multi modalities, context awareness, user modeling and learning capability are in their evolution state. For example accurate and time efficient algorithms for information fusion and extracting useful information for independent and inter dependent modalities are yet to be developed [30]. Similarly context awareness and user plan recognition (identification and estimating the user activity in a specific context) is a complex task to model computationally. Some pattern recognition algorithms and HMMs have been applied for predicting user activity but they have their own limitations [15]. Same holds true for incorporating user modeling and personalization capabilities into interfaces because they not only take user habits, skill set, interests, preferences etc into account but also the user personality, emotions, mood and behavior are taken into account for which there are no precise computational model and framework exists and they are presently research hotspots. The noteworthy contributions in the domain are listed as following:

In the ground breaking paper [27] T. Li, P. Yue, Y. Qiu. and G. Zhong reviewed the concepts and related work on emotions and personality in psychology and artificial intelligence and then presented a framework of learning companion agent with emotions and personality recognition. Personality is defined as the scientific study of human behavior and mental processes. The model that has got wide acceptance is the OCEAN (Openness, Conscientiousness, Extraversion, Agreeableness and Neuroticism) model of personality. These five factors collectively form the personality of an individual. Standard questionnaire and interaction sessions with users are arranged. The individual factors are computed considering the presence of respective low and high score traits. Values for all these five factors, in a predefined fixed range, are calculated according to the presence of these traits and a personality vector is finally framed for each user.

The OCC model is one of the mostly used models of emotion. In this model the emotions are captured by observing the user's sequence of actions, reactions to particular events, interaction behavior etc. Then these emotions are categorized into positive or negative type under a particular context. Some positive emotions are contentment, appreciation, love, happy, enjoy etc. and some negative emotions are dissatisfaction, shame, hate, fear anger etc. As per OCC model any emotional state is represented by 22 element vector. But practically 04 are considered in the proposed framework. The values for all these elements are calculated according to the present state of these emotions and an emotion vector is finally constructed for each user. This paper also discusses about the unavailability of widely accepted model for mood recognition.

The paper [28] applies three Neural Networks namely Multilayer Perceptron (MLP), Principal, Component Analysis (PCA), Generalized Feed Forward Neural Network (GFFNN) and then compares performance of these to recognize the human emotions from facial expressions. The result showed that MLP is most suitable for human emotion recognition from facial expressions using DCT. This paper aims at developing "Mind Implemented Robots" that can do intellectual conversation with human beings.

A new method for user modeling was proposed by $\mathrm{K}$. Leichtenstern, E. Andre [29] in their paper. In this method the diversity among users can be addressed using different personas. Each persona represents a group of similar type of perspective users and contains useful information about them. The persona consists of two parts

i. Mental Model: It contains the general information of intended users and helps to identify/build user's mental makeup along with positive attitude to use the proposed system.

ii. Main and Situational Goals: This includes the persona vise specifications of prime and sub objectives that the users may have in using the proposed system. For example while connected in online conferencing with the boss on very important issue stop other applications automatically which eat channel bandwidth.

Once all these personas have been identified, defined and computationally modeled, the interfaces are developed and evaluated in iterative fashion.

In the normal course of our interaction with others and surroundings, we use many receiving channels and many response channels, but when it comes to interact with computers a few channels are used. Definitely there is a room for additional modalities like speech and language processing, gesture and posture recognition, emotions and mood identification, computer vision, gaze tracking, lip reading, touch feeling, head and hand movement, eyebrow identification. The multimodal interaction fills the gap between multidimensional cognitive and functional space of human being and single dimensional information space of computers. 
Major challenge in using multimodal interfaces is the development of efficient and fast algorithms to collect and integrate the information in real time collected from various interaction channels. Multi interface agent architecture is practicable solution for this problem [30]. An Interface Agent (IA) is autonomous entity which deals with all aspects of communication through a single modality. Each agent performs pre/post processing and is enriched with learning and adaptive abilities. All such agents work in collaborative and coordinated fashion.

\section{PROPOSED METHODOLOGY}

Presently, and much more in the near future, the intelligent technology based systems powered with array of sensors connected in high speed network going to be indispensible assisting part of the normal human life activities, occupying the same space as humans, and performing the tasks in collaboration and cooperation with humans. The interaction interfaces, patterns, styles, methods of these intelligent systems with humans is very focal issue that must be considered while specifying, designing, developing and implementing these systems for this cooperative existence to be more effective, usable, acceptable and useful.

For this to achieve firstly a survey is to be conducted among computer users from different professional background and diversified fields in order to identify and understand the requirements, demands and expectations of the users of technological systems. If it is known priory that what is important from the user point of view and what characteristics the user expect in the interfaces then by incorporating this knowledge into interfaces we can definitely make them offering better user experience and more satisfying. This knowledge will be collected from users by firstly asking them to fill a questionnaire form then analyzing these forms. Once all these requirements, demands and expectations of the users have been clearly identified and understood they would be grouped on the similarity basis and co-relation among different groups is established if possible. After this those design principles and concepts would be determined which are, singly or collectively, capable enough to encompass and address all the identified requirements, demands and expectations of the users. This enables us to have consolidated conceptualization of problem space.

The next step is to find out and determine appropriate approaches, tools and concerned issues which put these design principles and concepts into practice and allow developing working models/prototypes. The development life-cycle of Computational System with Intelligent and Adaptive interfaces will consist of following steps:

Step1: Thorough investigation, identification, understanding and consolidated conceptualization of problem space i.e. specified set of users, their characteristics and skill set, usages requirements and patterns, practicing environment etc.

Step2: Analyze and build alternative prototype designs and interactive interfaces in such a way that make maximum use of above mentioned points.

Step3: Developing the working models and critically evaluate them throughout the development process in the light that what was to be developed and what is developed, The quality of user experience it offers etc.

Step4: Remove drawbacks if found and incorporates the suggestions to improve the interfaces.
Step5: Repeat the process from step-2 till satisfactory design in all respects is achieved.

At the last, the developed models/prototypes would be critically evaluated on the basis of various parameters like usability, ergonomics, consistency, contextual aid, the quality of user experience they offer etc. It will allows us to remove the drawbacks (if found) and incorporate the suggestions from evaluation process to improve the quality of interfaces and interactions.

\section{CONCLUSION AND EXPECTED OUTCOME OF PROPOSED WORK}

Human computer interaction along with intelligent and adaptive interface is very young field. Following outcomes are expected as a result of this work:

- Rich collection of properly filled questionnaire forms. The purpose of these forms is to collect the information about the requirements, demands and expectations of the users of technological systems. This information can also be used for many more research purposes in future.

-A working model of the futuristic intelligent and adaptive interfaces would be designed.

- The resulting concepts, principles and approaches for designing intelligent and adaptive interfaces of this work, singly and collectively, will lead towards significant improvements in following characteristics of interfaces and associated interaction patterns:

i.Usability.

ii. Quality of user experience along with user satisfaction.

iii. User friendliness and flexibility.

iv. Usefulness.

v. Adaptive behavior and personalization.

- This work will also propose an evaluation framework that can be used to assess the quality of the interfaces against parameters like usability, contextual aid etc.

-In future this work will pave for the researchers working in this domain.

\section{REFERENCES}

[1] Niitsuma M., Yokoi K., Hashimoto H.: Describing Human-Object Interaction in Intelligent Space. HSI 2009, pp 395-399. Catania, Italy, May 21-23, 2009.

[2] Kawahara, Y., Morikawa, H., Aoyama, T.: Intelligent Interface Systems Utilizing User Context-Awareness. Sixth International Conferences on Machine Learning and Cybernetics, pp. 2119-2124. Hong Kong, 19-22 August 2007.

[3] Seneler, C. O., Basoglu, N., Daim, T. U.: A Taxonomy for Technology Adaption: A Human Computer Interaction Perspective. PICMET Proceedings, pp. 2208-2219. Cape Town, South Africa, 27-31 July 2008.

[4] Ehlert, Patric, A.M.: Intelligent User Interface: Introduction and Survey. Research Report DKS03-01 / ICE 01, Version 0.9, Feb 2003.

[5] Sharp, H., Rogers, Y., Preece, J.: Interaction Design, Beyond Human Computer Interaction, Second Edition. Wiley India, 2007. 
[6] Yampolskiy, R. V.: Human Computer Interaction Based Intrusion Detection. Fourth International Conference on Information Technology, ITNG'07, pp. 837-842, 02-04 April 2007.

[7] Lester, C.Y.: Advancing the Multidisciplinary Nature of Human Computer Interaction in a Newly Developed Undergraduate Course. First International Conference on Advances in Computer Human Interaction, pp.177-182, 2008.

[8] Rusu, C., Rusu, V., Roncagliolo, S.: Usability Practice: The Appealing Way to HCI. 1st International Conference on Advances in Computer Human Interaction, pp. 265270, 2008.

[9] Zuffi, S., Brambilla, C., Beretta, G., Scala, P.: Human Computer Interaction: Legibility and Contrast. 14th International Conference on Image Analysis and Processing (ICIAP 2007), IEEE Computer Society 2007, ISBN 0-7695-2877-5, pp. 241-246. Modena, Italy, 1014 September 2007.

[10] Carroll, J.M.: Human-Computer Interaction, Second Impression. Pearson Education, 2008.

[11] Yang, Z. Li M., Zhou Y.: Embedment of 3D Virtual Human into Webpages for Visual Speech Synthesis Purpose. International Conference on Virtual Environments, Human-Computer Interfaces and Measurements Systems, VECIMS 2009. Hong Kong, China, May 11-13, 2009.

[12] Dearden, A., Howard, S.: Capturing user requirements and priorities for innovative interactive systems. Proceedings of Computer Human Interaction Conference, Australasian Volume, pp. 160-167, 30 Nov- 4 Dec 1998.

[13] Ozkan, N., Paris, C., SImpsom, B.: Towards an Approach for Novel Design. Australasian Computer Human Interaction Conference, pp 186-191. Adelaide, S. A., Australia, 30 Nov-4 Dec 1998.

[14] Goodman, B. A., Litman, D. J.: Plan Recognition for Intelligent Interfaces. 6th IEEE Conference on AI Applications (CAIA-90), pp. 297-303. Santa Barbara, March 1990.

[15] Sanchez, D., Tentori, M., Favela, J.: Hidden Markov Model for Activity Recognition in Ambient Intelligence Environment. 8th Mexican International Conference on Current Trends in Computer Science, pp. 33-40, 2007.

[16] Day, A.K.: Understanding and Using Contexts. Personal and Ubiquitous Computing Journal, Volume 5 (1), pp. 4-7, 2001 .

[17] Yoshida, K.: User Commands prediction by using graphbased induction. Sixth International Conference on Tools with Artificial Intelligence, pp. 732-735. New Orleans, LA, USA, 6-9 Nov 1994.

[18] Sutterer, M., Droeghorn, O., David, K.: UPOS: User Profile Ontology with Situation Dependent Preferences Support. First International Conference on Advances in Computer Human Interaction, pp.230-235, 2008.
[19] Arai F., Fukuda, T., Yamamoto, Y., Naito, T., Matsui, T.: Interactive Adaptive Interface Using Recursive Fuzzy Reasoning. Proceedings of IEEE Virtual Reality Annual International Symposium, VR '93, pp. 104-110. Seattle, Washington, USA, 18-22 September 1993.

[20] Bauckhage, C., Fritsch, J., Rohlfing, K. J., Wachsmuth, S., Sagerer, G.: Evaluating Integrated Speech- The Image Understanding. Proceedings of Fourth IEEE International Conference on Multimodal Interfaces (ICMI'02),ISBN: 0-7695-1834-6, pp. 9-14, 2002.

[21] Pirhonen, A.: Analysis of the Concept of Redundancy Concerning the Design of Multimodal Combinations of Output Elements. Proceedings of third Asia Pacific Computer and Human Interaction, pp. 273-278, 15-17 Jul 1998.

[22] Picard, R.W., Vyzas, E., Healey, J.: Towards Machine Emotional Intelligence: Analysis of Affective Physiological State. IEEE Transaction on Pattern Analysis and Machine Intelligence, Vol. 23, No. 10, Oct 2001.

[23] Okada, N., Inui, K., Tokuhisa, M.: Towards Affective Integration of Vision, Behavior and Speech Processing. Proceedings of the Integration of Speech and Image Understanding. pp 49—77, 1999.

[24] Oinas-Kukkonen, H., Harjumaa, M.: Towards Deeper Understanding of Persuasion in Software and Information Systems. First International Conference on Advances in Computer-Human Interaction, pp. 200 205, 10- 15 Feb, 2008

[25] Chang, E., Dillon, T., Calder, D.: Human System Interaction with Confident Computing: The Mega Trend. Conference on Human System Interactions, pp. 1-11. Krakow, Poland, 25-27 May 2008.

[26] Liu J., Ando H.: Emotion Eliciting and Decision Making by Psychodynamic Appraisal Mechanism. HIS 2008, Kralow, Poland, May 25-27, 2008.

[27] Li, T., Qiu, Y., Yue, P., Zhong, G.: Exploiting Model of Personality and Emotion of Learning Companion Agent. IEEE/ACS International Conference on Computer Systems and Applications (AICCSA 2007), pp. 860865. Amman, Jordan, 13-16 May 2007.

[28] Kharat G. U. , Dudul S. V. : Emotion Recognition from Facial Expression Using Neural Networks. HSI 2008, Krakow, Poland, May 25-27, 2008.

[29] Leichtenstern, K., Andre, E.: User Centered Development of Mobile Interfaces to a Pervasive Computing Environment. First International Conference on Advances in Computer Human Interaction, pp.114119, 2008.

[30] Zhu, Z., Wang, J.: Multi-Agent Based Approach to Support HCI. 5th International Conferences on Machine Learning and Cybernetics, pp. 188-191, Dalian,13-16 August 2006. 